\title{
La comunicación noviolenta entre teoría y práctica. Una revisión sistemática
}

\author{
Nonviolent communication between theory and practice. A systematic review
}

Alina Danet Danet

Departamento de Organización de Empresas, Marketing y Sociología - Universidad de Jaén adanet@ujaen.es

\section{Resumen}

El objetivo de esta revisión fue analizar el uso y aplicación de la comunicación noviolenta en el ámbito científico-académico internacional. Se realizó una búsqueda bibliográfica de los estudios publicados desde 2010 en PubMed, Scopus y Google Académico. Se seleccionaron 23 artículos que se revisaron en dos fases: analítico descriptiva y de síntesis narrativa. Se identificó la heterogeneidad geográfica, disciplinar y metodológica en la aplicación de la comunicación noviolenta. Desde las Ciencias de la Educación, diez ensayos y estudios empíricos consideraron la CNV como paradigma útil para crear comunidades educativas inclusivas y fomentar la integración, colaboración y empatía, en base al desarrollo de competencias intra e interpersonales. En Ciencias de la Salud, Ciencias Sociales y ámbito multidisciplinar, los estudios de intervención, la mayoría con diseño cuasi-experimental, mostraron el impacto positivo de las estrategias formativas de comunicación noviolenta sobre la empatía, gestión del conflicto, prevención y abordaje de la violencia y delincuencia, la salud emocional y la calidad de las relaciones familiares, interprofesionales y comunitarias. Los hallazgos situaron la comunicación noviolenta dentro de un marco teórico humanista y, a nivel práctico, evidenciaron sus beneficios para mejorar las vivencias personales, la interacción y convivencia entre individuos. Se necesitaría fomentar diseños metodológicos sólidos para reforzar, desde ámbitos científico- académicos, los resultados basados en la evidencia sobre el impacto de la comunicación noviolenta y su aplicación.

Palabras clave: Competencias comunicativas, Habilidades de comunicación, Educación emocional, Métodos, Análisis narrativo, Publicación científica, Interdisciplinariedad, Ciencias Sociales

\section{Abstract}

The objective of this revision was to analyze the use and application of Nonviolent Communication in scientific and academic international contexts. The bibliographic search in PubMed, Scopus y Google Scholar identified 23 articles published from 2010 onwards. The process of revision included two phases: an analytical description, followed by a narrative synthesis. The revision revealed the heterogeneity of geographical contexts, disciplines and methodologies used. In Education, ten essays and empirical studies considered Nonviolent Communication as a useful paradigm with positive effects on creating inclusive educational communities and fostering integration, collaboration and empathy, by developing intra and interpersonal competencies. In Health and Social Sciences, as well as from multidisciplinary approaches, interventional studies, most of them with quasi-experimental design, proved the positive impact of Nonviolent Communication training. Most important impact dimensions were empathy, conflict management, prevention and control of violence and delinquency, as well as socio-cultural variables, including 
aspects of emotional health and quality of family, work and community relationships. These findings supported Nonviolent Communication as a Humanistic theoretical framework and, on a practical level, described its contribution to the improvement of personal experiences, interaction and social coexistence. In order to enhance the scientific evidence over the impact of Nonviolent Communication and its application, further research using robust methodological design is needed.

Keywords: Communication competences/skills, Communication skills, Emotional education, Methods, Narrative Analysis, Scientific publication, Interdisciplinarity, Social Sciences 


\section{Introducción}

La evolución es tan creativa... Así es como tenemos jirafas (Kurt Vonnegut).

La jirafa es el mamífero terrestre con el corazón más grande -inspira compasión y generosidad - y el cuello más largo —evoca amplitud de miras - $y$, aunque no tiene cuerdas vocales, en los años 60, fue elegido por el psicólogo estadounidense Marshall B. Rosenberg (1934-2015) como símbolo de la comunicación noviolenta (CNV), también conocida como lenguaje de la jirafa, comunicación compasiva o, menos frecuente, comunicación colaborativa (Leu, 2003).

En su libro fundacional "Nonviolent Communication. A Language of Life", Rosenberg (2003) define la CNV como "una manera de comunicarnos que nos lleva a dar desde el corazón, a conectarnos con nosotros mismos y con otras personas" (p.18). Enraizada en la Psicología Humanista y en el concepto gandhiano de compasión, -definido como renuncia a la violencia y, a la vez, fuerza para el cambio social (Gandhi, 1971)-, la CNV se integra dentro del paradigma de la noviolencia, que López Martínez (2015) define como "una metodología, una doctrina éticopolítica y una manera de construir la paz que se orienta hacia una filosofía coherente que busca un amor por el conocimiento, la experiencia y la vida".

Siguiendo al mismo autor, la noviolencia es un concepto complejo y la búsqueda de consenso sobre sus principios y valores esenciales, reflejada en el análisis de diversas experiencias históricas (López Martínez, 2000) se podrían sintetizar en: 1 . Negarse a matar o preservar una vida digna, entendido como un derecho de los seres humanos que implica reducir al máximo el uso de la violencia, no sólo a nivel interpersonal sino también institucional; 2. Buscar la verdad, como una experiencia y predisposición vital, a favor de la tolerancia y la renuncia a disponer la verdad absoluta; 3. Generar diálogo y escucha activa, como actividad de exploración intra e interpersonal, basada en la empatía y el humanismo; 4. Buscar modos alternativos $\mathrm{y}$ creativos de pensamiento, deslegitimando las modalidades violentas o reactivas y apostando por la apertura y optimismo antropológico (López Martínez, 2015).

El marco normativo de noviolencia permite desarrollar métodos de intervención y gestión pacíficos del conflicto, encontrar fórmulas alternativas para oponerse a las injusticias y la opresión, humanizar los procesos y relaciones socio-políticas y culturales, así como favorecer la introspección personal y la integración de la humanidad con la naturaleza (López Martínez, 2015).

Desde esta óptica de reencuentro entre pensar y sentir, entre racionalidad y sensibilidad (López Martínez, 2015), la CNV parte de considerar que todas las personas tenemos las mismas necesidades que, una vez satisfechas, generan sentimientos de alegría, contento y paz y, en caso contrario, enfado, miedo, frustración o vergüenza. Por otra parte, para cubrir necesidades, empleamos estrategias diferentes, cargadas de condicionamientos socio-culturales, de las cuales a menudo no somos conscientes y que, por tanto, dificultan la interacción comunicacional (Rosenberg, 2003).

Con el objetivo de crear conexiones interpersonales basadas en respeto y empatía, que faciliten la resolución de conflictos, la CNV propone la expresión honesta y escucha empática de las necesidades propias y ajenas, y para ello, estructura un proceso de cuatro pasos básicos: Observación, Sentimientos, Necesidades y Peticiones. 
La Observación supone describir claramente y sin interpretar, los hechos y situaciones que nos afectan. En este paso, es importante diferenciar la observación de evaluación, juicios o críticas. La segunda fase requiere identificar y expresar estados internos, emociones o sentimientos que nos genera la situación observada, distinguiéndolos de pensamientos, creencias $\mathrm{u}$ opiniones. En el tercer paso, se definen las Necesidades, que representan valores o principios universales en todos los seres humanos, son transculturales y no pueden estar delimitadas en tiempo o espacio, ni supeditadas a personas, objetos o acciones concretas (en cuyo caso serían estrategias, no necesidades). Por último, al formular las Peticiones, se transmite de manera precisa, realista, afirmativa y negociable lo que deseamos, sin imponer, lo que contrapone las peticiones a las demandas o exigencias.

Los principios de la CNV se han aplicado a nivel mundial desde diferentes perspectivas y en diversos contextos. En el ámbito educativo se señaló la utilidad de la CNV para consolidar el modelo con enfoque colaborativo (Koegel, 2002; Jones, 2009), facilitar la conexión, confianza y receptividad (Cox y Dannahy, 2005) o mejorar las habilidades de comunicación, empatía y bienestar emocional del profesorado (Hooper, 2015). A nivel sanitario, se han documentado los beneficios de la CNV para aumentar el nivel de empatía del personal médico y enfermero (Rosenberg y Molho,1998; Sears, 2013; Chung y Kim, 2011). En la prevención y abordaje de la violencia y resolución de conflictos, la CNV ha tenido aplicabilidad en contextos penitenciarios y de intervención policial (Shoemaker, 2004; Dougan, 2011; Nash, 2007), así como en situaciones de tensión política, interétnica o comunitaria (Dzaferovic, 2012; Kök, 2009).

Pese al creciente interés de las Ciencias Sociales y de la Comunicación o de las Humanidades, la literatura disponible no es concluyente con respecto a los resultados, evaluación e impacto de la CNV a nivel científico y académico. Especialmente hay carencias en torno a estudios empíricos $\mathrm{y}$ experimentales (Sears, 2013) y no se ha consolidado una línea conceptual y teórica clara para la aplicación de la $\mathrm{CNV}$ en diferentes contextos de intervención.

Hasta la fecha, en el contexto occidental, no se han sistematizado los resultados sobre el uso y aplicación de la CNV, a diferencia de otros contextos, como el oriental (Yang y Kim, 2016). Pretendiendo responder a esta necesidad, el objetivo general de esta revisión bibliográfica es identificar y analizar el uso y aplicación de la CNV en el ámbito científicoacadémico internacional.

Como objetivos específicos se establecen: 1. Cuantificar, localizar y describir a nivel geográfico y disciplinario los estudios que usan la $\mathrm{CNV}$; 2. Analizar los marcos teóricos y metodológicos empleados en el uso de la CNV; y 3. Sintetizar la aplicación de la $\mathrm{CNV}$, sus resultados e impacto, en diferentes campos de estudio.

\section{Material y métodos}

Se realizó una revisión sistemática cualitativa de artículos publicados entre 2010 y 2018 en las bases de datos PubMed y Scopus y en el buscador Google Académico. Los descriptores y términos de búsqueda empleados se presentan en la Figura 1. 


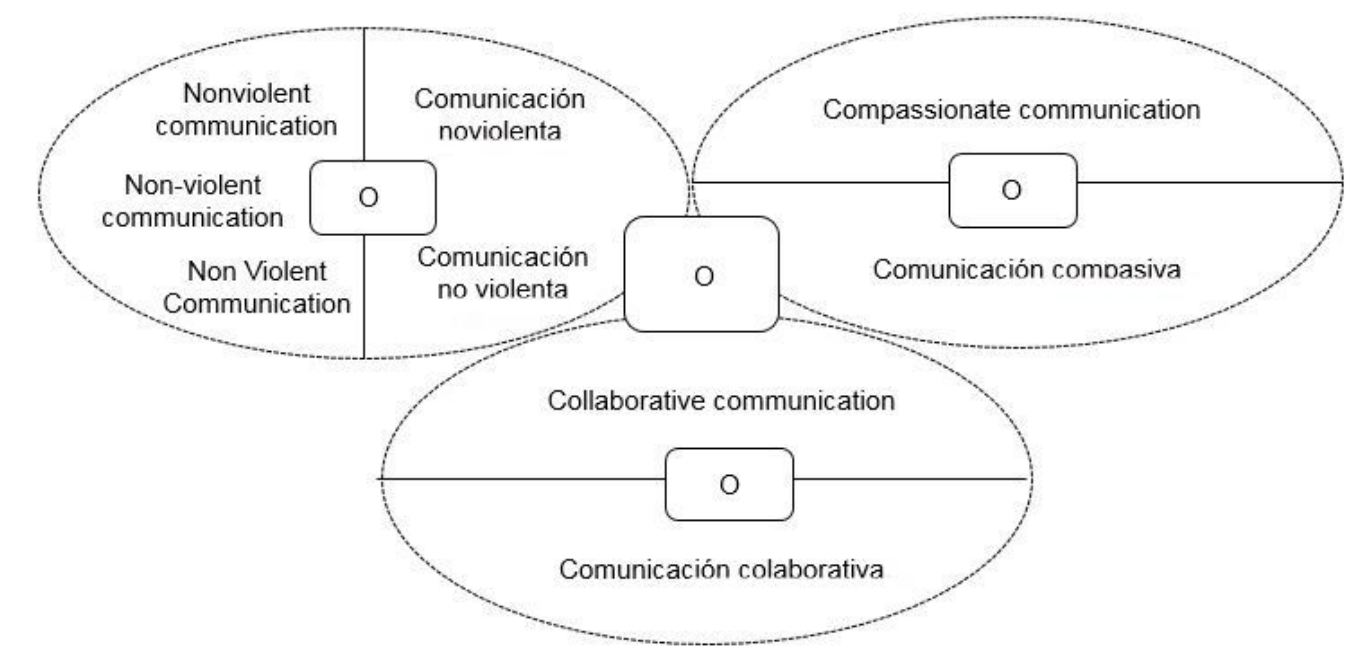

FIGURA 1. DESCRIPTORES Y TÉRMINOS DE BÚSQUEDA EN ESPAÑOL Y EN INGLÉS

Los criterios de inclusión y exclusión se definieron en base a las siguientes categorías:

- Concepto de CNV. Inclusión: uso del término y aplicación de CNV definida por Rosenberg (2003). Exclusión: CNV como estrategia de recogida de datos en investigación cualitativa (Bourdieu, 1999) o en el abordaje de patrones lingüísticos (Mikiloc, 2016).

- Tipo de fuentes: Inclusión: primarias. Exclusión: Fuentes secundarias (artículos de revisión, monografías) y terciarias (folletos, libros de texto, manuales, guías), literatura gris, editoriales, cartas al director, actas de congresos.

- Revisión por pares. Inclusión: sí. Exclusión: no.

- Idioma de publicación. Inclusión: inglés o castellano. Exclusión: otros idiomas.

- Fecha de publicación. Inclusión: 2010-2018. Exclusión: anterior a 2010.

El proceso de identificación, selección, elección e inclusión de las fuentes se describe en la Figura 2.

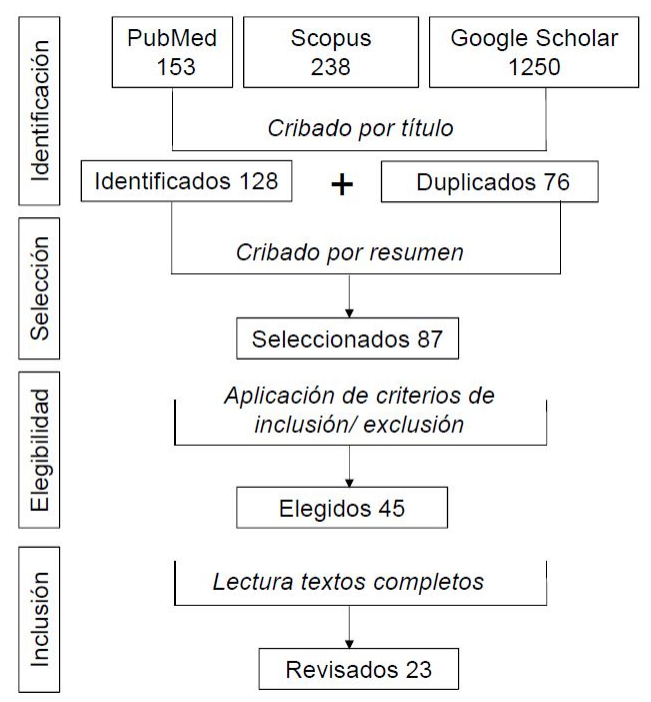

FIGURA 2. PROCESO DE SELECCIÓN DE LAS FUENTES 
El proceso de revisión se realizó en dos fases. En la primera, analítico-descriptiva, se extrajeron y estructuraron: país, ámbito/ disciplina, diseño, metodología, marco teóricoconceptual, resultados y conclusiones de las fuentes. Para definir la categoría ámbito/ disciplina, se tuvo en cuenta la afiliación académica y la especialidad de las personas firmantes, así como el campo de indexación de las revistas.

En la segunda fase, de síntesis narrativa, se identificaron y analizaron las principales temáticas y dimensiones emergentes, a partir del método inductivo de análisis de contenido (Finfgeld-Connett, 2014).
Para evaluar los aspectos de calidad, ética y validez metodológica se usaron la declaración ENTREQ (Tong et al, 2012) y el protocolo PRISMA-P (Shamseer et al., 2015).

\section{Análisis y resultados}

Se revisaron 23 estudios con dispersión geográfica. La figura 3 representa la distribución por ámbitos y disciplinas, así como localización, con mayor concentración en el continente norteamericano (14 estudios) y europeo (5 estudios).

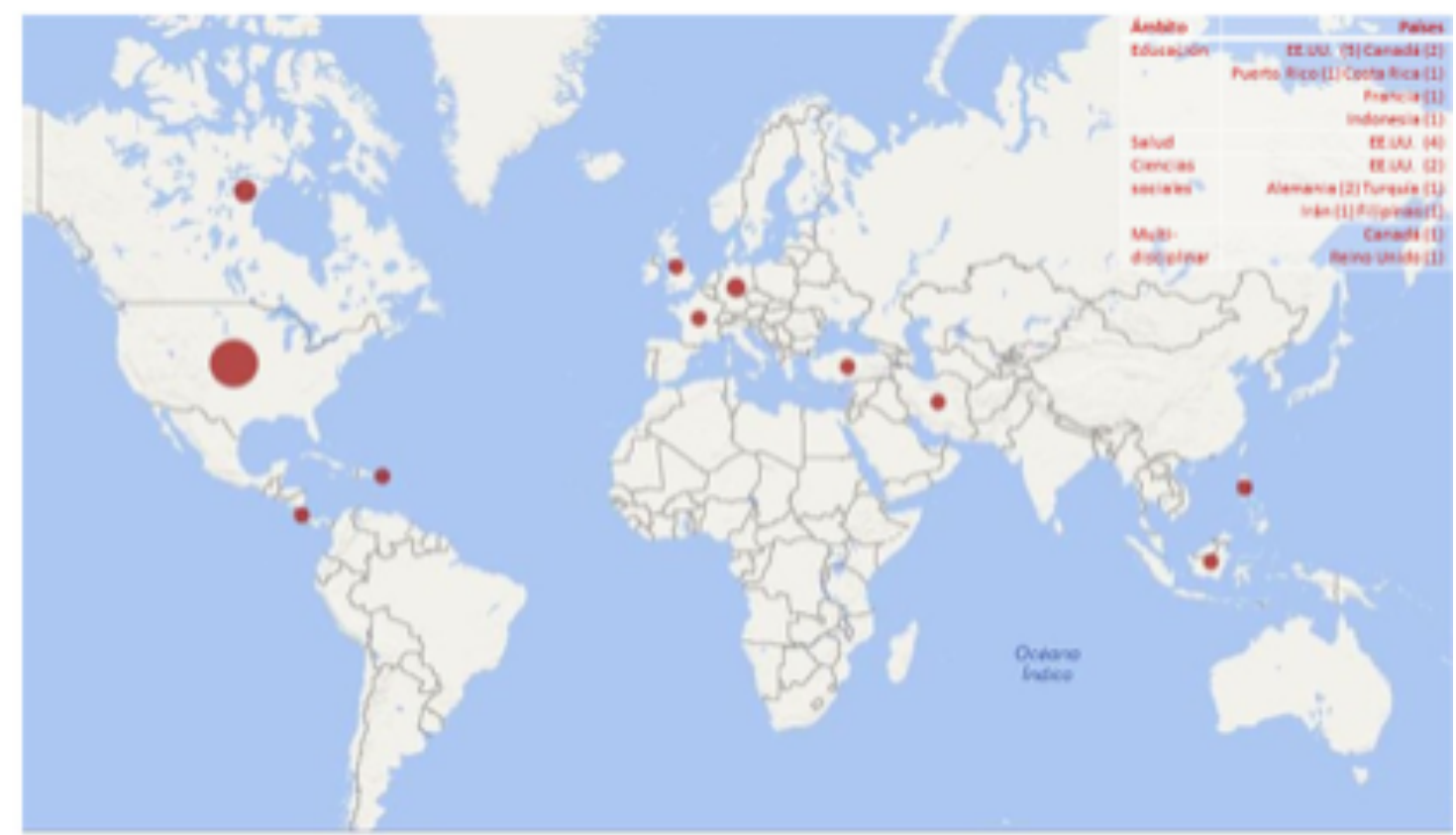

FIGURA 3. DISTRIBUCIÓN GEOGRÁFICA Y DISCIPLINAR DE LOS ESTUDIOS

\subsection{La CNV en Ciencias de la Educación}

En el marco académico de las Ciencias de la Educación se encontraron 10 estudios, de los cuales 8 con origen americano- 4 de EE.UU. (Agnew, 2012; Baesler, 2017; Frey, 2017; Hao, 2017), 1 de EE.UU. y Canadá (Baesler y Lauricella, 2014), 1 de Canadá (Schwind et al,
2016), 1 de Costa Rica (Madrigal y Vargas, 2014), 1 de Puerto Rico (Torres, 2013)-, 1 europeo (Albe y Gombert, 2012 en Francia) y 1 asiático (Qudsi, 2018 en Indonesia).

La Pedagogía (2), Didáctica (4) y Planificación curricular (3) concentraron la mayor parte de las publicaciones revisadas, mientras que, por el tipo de estudio, se observó una distribución equilibrada entre 5 estudios 
empíricos descriptivos y 5 ensayos. La población diana de los estudios la representaron principalmente estudiantes universitarios de pregrado (7 artículos), aunque también estudiantes de Bachillerato (1 estudio) y de Educación Primaria (1 estudio).

En el campo de la Pedagogía, el ensayo de Hao (2017) se apoyó en la CNV para la creación del modelo de "pedagogía crítica compasiva", situado teóricamente en la pedagogía crítica de la comunicación (Fasset y Warren, 2007). Dirigida al alumnado universitario de primera generación (sin antecedentes familiares conocidos de acceso a la Universidad), la aplicación de la CNV contribuyó a desarrollar una pedagogía culturalmente inclusiva, entendida como proceso de interacción intersubjetiva, y a mejorar la adaptación pedagógica a las necesidades específicas del alumnado, minimizar la presencia de estereotipos y prejuicios culturales e incentivar la autoreflexión docente. En este sentido, el propio autor confirmaba que la CNV hacía posible una "pedagogía del bienestar" ("feel good pedagogy"), no sólo para el alumnado sino también para el docente (Hao, 2017:98).

Por otra parte, desde Puerto Rico, Torres (2013) presentó en su ensayo, un enfoque sistémico de aplicación de la CNV desde perspectiva teórica y práctica. La autora, con formación en Ciencias de la Educación, sintetizó diversas teorías socio-educativas sobre la violencia (Bernstein, 1996; Freire, 2004; Vygotsky, 1982; Bandura, 1973), para concluir que las teorías provenientes de la Sociología, Psicología y Filosofía, sustentan la implementación de estrategias educativas basadas en CNV orientadas a mejorar la comunicación y aprendizaje colaborativo en el aula.

Desde el ámbito de la Didáctica y a partir de la experiencia universitaria en EE.UU., dos ensayos analizaron la aplicación de la CNV en la didáctica de la religión (Agnew, 2012) y de la literatura (Frey, 2017). Elizabeth Agnew (2012) se apoyó en el marco teórico de la ética de la conversación (Chopp, 2005) y partió del paralelismo clase-comunidad, en cuanto grupos con prácticas sociales compartidas (MacIntyre, 2006), para concluir que la CNV favorece la escucha activa, la expresión de las necesidades individuales y la resolución de conflictos en el aula. Además, la CNV facilitó la reflexión y el debate, redujo los posicionamientos antagónicos y ayudó a identificar empáticamente las necesidades de todo el alumnado.

Por otra parte, Renea Frey (2017) presentó en su ensayo sobre didáctica de la literatura, el diseño y aplicación del curso "Escritura como acción social" en una universidad privada jesuita. Al amparo del paradigma de eloquentia perfecta (Gannet y Brereton, 2016) y de los principios sociopolíticos de la retórica (Booth, 2004; Ratcliffe, 2005), la autora argumentó que la práctica de CNV favorece la escucha profunda, el desarrollo de la empatía y la vivencia corporeizada de la comunicación oral.

También en el ámbito de la Didáctica y centrándose en los beneficios de la Educación emocional (Bisquerra, 2003), las costarricenses Madrigal y Vargas (2014) expusieron su estudio de caso, para concluir que la aplicación de la CNV como recurso didáctico motivacional mejoró tanto la autoestima y autorrealización, como la comunicación y empatía de los participantes en un programa de formación de inglés como idioma extranjero. Citando a las autoras, los resultados directos de la CNV generaron "un avance significativo de la gramática", a la vez que sentimientos de orgullo y satisfacción personal, "sentirse feliz acerca del progreso" (Madrigal y Vargas, 2014:331). 
Dentro del ámbito disciplinario de Diseño, desarrollo y evaluación curricular, 3 estudios de contexto norteamericano y 1 de Indonesia mostraron que la formación específica o transversal y la práctica de la CNV mejoran la empatía y la calidad relacional en diferentes niveles educativos.

En una colaboración interuniversitaria EE.UU.- Canadá, Baesler y Lauricella (2014) trabajaron en la creación del curso "CNV y paz”, partiendo de principios de enseñanza de la paz y de la vivencia de la espiritualidad en las aulas (McCarthy, 2002). En su publicación común, los autores exponen el diseño de la estrategia formativa, presentando dos estudios piloto y los resultados de la evaluación realizada con estudiantes de las dos universidades. Posteriormente, Beasler (2017) discutió en su ensayo de carácter autoetnográfico, el impacto de la educación para la paz dirigida a estudiantes residentes en una zona militarizada de EE.UU. Los resultados de los trabajos mostraron cómo los cursos de CNV amplían la visión del alumnado sobre el concepto de "paz", mejoran el conocimiento de modelos y personalidades referente en el activismo pacifista, favorecen el uso del lenguaje, las actitudes y comportamientos pacíficos, el pensamiento crítico y el compromiso social y con el entorno local, a la vez que aumentan la empatía.

Otra experiencia de diseño y pilotaje curricular la proporcionaron las canadienses Schwind, McCay, Metersky y Martin (2016) que describieron la implementación de un curso de comunicación terapéutica avanzada, con aplicación de la CNV a la formación interdisciplinar de pregrado. El marco referencial integró educación interprofesional en salud (D'Amour y Oandasan 2005), comunicación terapéutica como proceso empático (Younis, Mabrouk y Kamal 2015) y proceso narrativo reflexivo (Schwind, 2016).
Las fases seguidas para el diseño curricular fueron: 1. Identificación de principales competencias interprofesionales (se identificaron 9 competencias de práctica y de contexto, a partir de una revisión bibliográfica y la validación por métodos de consenso); 2 . Desarrollo de marco conceptual (se definieron: enfoque relacional de la comunicación terapéutica, enfoque centrado en la persona, colaboración en equipos interdisciplinares, evidencia en habilidades de comunicación terapéutica); 3. Diseño curricular (definición de contenido e implantación); y 4. Pilotaje. Las estrategias de aprendizaje propuestas fueron la lectura interpretativa y debate en grupo, terapia narrativa, terapia dialéctica conductual, entrevista motivacional, terapia de aceptación y compromiso y simulación de situaciones prácticas. La evaluación cualitativa reveló el impacto positivo del curso sobre el conocimiento, respeto y necesidad de colaboración interprofesional, ilustrado en los siguientes verbatims extraídos de las entrevistas realizadas: "Poder trabajar con y escuchar a otros profesionales de la salud fue realmente una experiencia útil, interesante $\mathrm{y}$ esclarecedora". "La colaboración es clave en la seguridad de los pacientes" (Schwind, McCay, Metersky y Martin, 2016:596).

Por último, un estudio descriptivo llevado a cabo en Indonesia (Qudsi, Trmulyaningsih, Novitasari y Stueck, 2018), explicó el diseño, pilotaje y evaluación de un módulo didáctico de $\mathrm{CNV}$ en educación primaria. Presentando como un proyecto de diseño de investigación y desarrollo aplicado a la educación (Gall et al 2003), y fundamentado como parte del proyecto "Escuela de Empatía Indonesia" (Stueck, 2010), la estrategia formativa específica en $\mathrm{CNV}$ tuvo carácter lúdico e incluyó: Introducción adaptada al público infantil a través de un cuento cuyo personaje es una jirafa; Observación y 
sentimientos; Observación y necesidades; Necesidades versus estrategias; Demandas y peticiones; y Afrontamiento de la ira. El curso mostró su eficacia para prevenir la violencia y mejorar la expresión emocional, aunque también puso de manifiesto la necesidad de adaptación de contenidos y prácticas al contexto socio-cultural local.

Dentro del ámbito de las Tecnologías de la Educación, Albe y Gombert, 2012 presentaron su proyecto de investigación basada en el diseño, a partir de una intervención didáctica con 15 estudiantes de bachillerato (17-18 años). Explicando la argumentación y búsqueda de consenso a través de la noción de habla exploratoria (Mercer 1996) y virtudes comunicativas
(Burbules y Rice 1991), la propuesta práctica en el aula se basó en juego de roles y la simulación de conferencia sobre calentamiento global. La puesta en práctica de los principios teóricos de CNV favoreció la exploración y el desarrollo de estrategias de argumentación, evitó la polarización de participantes en "ganadores" y "vencidos" y ayudó a regular la interacción interpersonal y gestionar el conflicto.

La Figura 4 esquematiza las aportaciones de los 10 estudios procedentes de las Ciencias de la Educación. Los trabajos se organizan según disciplinas y se sintetizan los principales resultados referidos a las aportaciones $y$ beneficios de aplicación de la CNV.

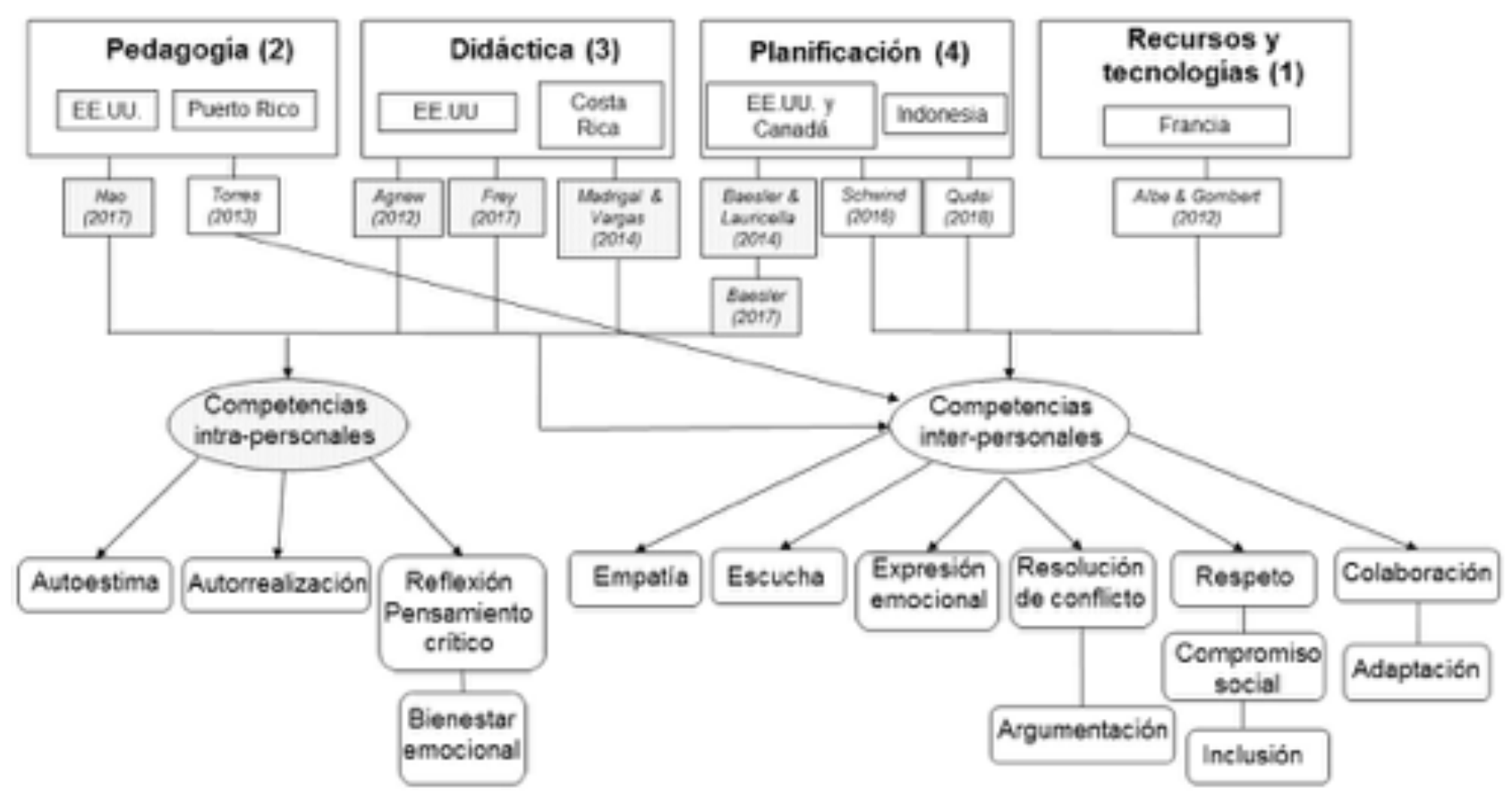

FIGURA 4. LA CNV EN CIENCIAS DE LA EDUCACIÓN: (SUB)DISCIPLINAS E IMPACTO EN COMPETENCIAS

3.2. La CNV en Ciencias de la Salud, Ciencias Sociales y ámbito multidisciplinar

De los 13 estudios realizados fuera del ámbito educativo, 10 fueron estudios de intervención para evaluar el impacto de estrategias formativas específicas de CNV. De ellos, 8 tuvieron diseño cuasi-experimental. La Tabla 1 describe la metodología, estrategia formativa y evaluación de los estudios empíricos revisados.

Los otros 3 estudios no experimentales, fueron: estudio de caso sin intervención 
formativa (Boothe, Frasier, Weaver y WhiteKiehle, 2018), ensayo basado en investigación cualitativa (Burgon, Di Gammage y Hebden y ensayo teórico con estudio piloto $\sin$ evaluación (Nosek, 2012).

\section{TABLA 1. ESTUDIOS EMPÍRICOS DE EVALUACIÓN DEL IMPACTO DE ESTRATEGIAS FORMATIVAS} EN CNV.

\begin{tabular}{|c|c|c|c|c|}
\hline Estudio & Diseño & Participantes & $\begin{array}{l}\text { Intervención formativa } \\
\text { (Estructura y contenidos) }\end{array}$ & $\begin{array}{l}\text { Evaluación (instrumentos } \\
\text { y técnicas) }\end{array}$ \\
\hline $\begin{array}{l}\text { A } 1 \text { s h u g h ry } \\
(2018)\end{array}$ & $\begin{array}{l}\text { I } \mathrm{n} \text { form e } \mathrm{de} \\
\text { campo }\end{array}$ & $\begin{array}{l}10 \text { mujeres sirias } \\
\text { de centro de } \\
\text { a cogida para } \\
\text { p e r s o n a s } \\
\text { refugiadas }\end{array}$ & $\begin{array}{l}2 \text { sesiones formativas y } \\
\text { seguimiento bisemanal } \\
\text { durante un mes. } \\
\text { Principios básicos de CNV } \\
\text { aplicados a través de técnicas } \\
\text { del Teatro del Oprimido } \\
\text { (Boal, 2005) }\end{array}$ & $\begin{array}{l}\text { C u e stio n a rio d e } \\
\text { elaboración propia con } \\
\text { preguntas abiertas }\end{array}$ \\
\hline $\begin{array}{l}\text { A } 1 \mathrm{t} \text { m a n } \\
\text { Schönefeld } \mathrm{y} \\
\text { Rorth }(2015)\end{array}$ & $\begin{array}{l}\mathrm{C} u \mathrm{a} \mathrm{s} \text { - } \\
\text { experimental } \\
\text { cuantitativo } \\
\text { aleatorizado } \\
\text { c on grupo } \\
\text { control }\end{array}$ & $\begin{array}{l}172 \text { profesionales } \\
\text { de Enfermería en } \\
\text { prácticas }\end{array}$ & $\begin{array}{l}4 \text { días consecutivos. } \\
\text { Empatía y el cortocircuito } \\
\text { empático, Principios básicos } \\
\text { de CNV, Aplicación de la } \\
\text { CNV, Role-play }\end{array}$ & $\begin{array}{l}\text { Cuestio n a rio de } \\
\text { competencia emocional, } \\
\text { Cuestio nario de } \\
\text { regulación emocional, } \\
\text { Escala de irritación, } \\
\text { Escala de implicación, } \\
\text { Inventario de Síntomas }\end{array}$ \\
\hline $\begin{array}{l}\text { Marlow, } \\
\text { Nyamathi, } \\
\text { Grajeda, } \\
\text { Bailey, Weber y } \\
\text { Younger (2012) }\end{array}$ & $\begin{array}{l}\text { Cuasi- } \\
\text { experimental } \\
\text { mixto. } \\
\text { Investigación } \\
\text { participativa } \\
\text { basada en la } \\
\text { comunidad. }\end{array}$ & $\begin{array}{l}30 \text { internos } \\
\text { penitenciarios }\end{array}$ & $\begin{array}{l}8 \text { sesiones de } 1 \text { hora. } \\
\text { Introducción, Sentimientos y } \\
\text { necesidades, Sentimientos } \\
\text { versus pensamientos, } \\
\text { Observación versus } \\
\text { evaluación, Círculo de } \\
\text { pensamientos, Empatía y } \\
\text { auto-expresión, Petición de } \\
\text { acciones, Rabia y gratitud }\end{array}$ & $\begin{array}{l}\text { Escala de Empatía } \\
\text { Emocional } \\
4 \text { Grupos focales }\end{array}$ \\
\hline $\begin{array}{l}\text { Museux, } \\
\text { Dumont, } \\
\text { Careau y Milot, } \\
\text { 2016) }\end{array}$ & $\begin{array}{l}\text { Cuasi- } \\
\text { experimental } \\
\text { cuantitativo. }\end{array}$ & $\begin{array}{l}9 \text { trabajadores } \\
\text { sociales, } \\
\text { educadores y } \\
\text { psico-educadores } \\
\text { de un centro de } \\
\text { asistencia socio- } \\
\text { sanitaria }\end{array}$ & $\begin{array}{l}1 \text { sesión de } 7 \text { horas. } \\
\text { Principios básicos de CNV, } \\
\text { comunicación y empatía. }\end{array}$ & $\begin{array}{l}\text { Herramientas de medición } \\
\text { en observación } \\
\text { participativa }\end{array}$ \\
\hline $\begin{array}{l}\text { ( N a fis e y } \\
\text { Ghazal, 2018) }\end{array}$ & $\begin{array}{l}\text { Cuasi- } \\
\text { experimental } \\
\text { cuantitativo } \\
\text { aleatorizado con } \\
\text { grupo control }\end{array}$ & $\begin{array}{l}15 \text { mujeres } \\
\text { adolescentes de } \\
\text { zonas } \\
\text { desfavorecidas de } \\
\text { Teheran }(+15 \text { en } \\
\text { grupo control). }\end{array}$ & $\begin{array}{l}8 \text { sesiones } \\
\text { Principios de CNV }\end{array}$ & $\begin{array}{l}\text { Escala de intencionalidad } \\
\text { suicida, Inventario de } \\
\text { estrategias de } \\
\text { afrontamiento ante } \\
\text { situaciones estresantes }\end{array}$ \\
\hline $\begin{array}{l}\text { Nosek y Durán } \\
(2017)\end{array}$ & $\begin{array}{l}\text { Estudio } \\
\text { cualitativo. } \\
\text { Investigación } \\
\text { participativa } \\
\text { basada en la } \\
\text { comunidad }\end{array}$ & $\begin{array}{l}20 \text { adultos y } 18 \\
\text { jóvenes }(9-17 \\
\text { años) de origen } \\
\text { latino residentes } \\
\text { en California }\end{array}$ & $\begin{array}{l}4 \text { sesiones para adultos y } 4 \\
\text { para jóvenes. } \\
\text { Introducción, Principios } \\
\text { básicos de CNV, Práctica de } \\
\text { CNV y aplicación a nivel } \\
\text { familiar }\end{array}$ & Grupos focales \\
\hline
\end{tabular}




\begin{tabular}{|c|c|c|c|c|}
\hline $\begin{array}{l}\text { Nosek, Gifford } \\
\text { y K o b e r } \\
(2014)\end{array}$ & $\begin{array}{l}\text { Cuasi- } \\
\text { experimental } \\
\text { mixto. }\end{array}$ & $\begin{array}{l}74 \text { estudiantes } \\
\text { universitarios de } \\
\text { primer curso de } \\
\text { Enfermería }\end{array}$ & $\begin{array}{l}3 \text { sesiones de } 2 \text { horas, } 1 \text { hora y } \\
45 \text { minutos. } \\
\text { Expresar sentimientos, } \\
\text { necesidades y peticiones. } \\
\text { Entender los sentimientos y } \\
\text { necesidades de otras personas }\end{array}$ & $\begin{array}{l}\text { Índice de Reactividad } \\
\text { Personal } \\
\text { Análisis de contenido de } \\
\text { diarios online } \\
6 \text { Grupos focales post- } \\
\text { intervención y } 2 \text { grupos } \\
\text { focales a los } 2 \text { años de la } \\
\text { intervención }\end{array}$ \\
\hline $\begin{array}{l}\text { Suárez, Lee, } \\
\text { Rowe, Gomez, } \\
\text { Murowchick y } \\
\text { Linn (2014) }\end{array}$ & $\begin{array}{l}\text { C u a s i - } \\
\text { experimental } \\
\text { mixto } \\
\text { estudios) }\end{array}$ & $\begin{array}{l}\text { Estudio } 1: 885 \\
\mathrm{i} \mathrm{n} \text { e } \mathrm{r} n \text { o } \mathrm{s} \\
\text { penitenciarios. } \\
\text { Estudio } 2: 13 \\
\text { internos }(+13 \text { en } \\
\text { grupo control) }\end{array}$ & $\begin{array}{l}2 \text { días }+3 \text { sesiones mensuales } \\
\text { de } 3 \text { horas } \\
\text { Principios de CNV y } \\
\text { mindfulness }\end{array}$ & $\begin{array}{l}\text { Estudio 1: Análisis } \\
\text { estadístico de tasa de } \\
\text { reincidencia } \\
\text { Estudio 2: } \\
\text { Inventario de Síntomas, } \\
\text { Escala de Atención Plena, } \\
\text { Escala de autocompasión } \\
\text { y Test de Ira y } \\
\text { Agresividad } \\
\text { Análisis de contenido de } \\
\text { juegos de rol }\end{array}$ \\
\hline $\begin{array}{l}\text { Vazhappilly y } \\
\text { Reyes (2017) }\end{array}$ & $\begin{array}{l}\text { Cuasi- } \\
\text { experimental } \\
\text { cuantitativo }\end{array}$ & 12 parejas & $\begin{array}{l}\text { Estructura: } 6 \text { sesiones en } 3 \\
\text { semanas. } \\
\text { Contenido: Conocimiento, } \\
\text { observación, sentimientos, } \\
\text { cambio, perdón, aprecio }\end{array}$ & $\begin{array}{l}\text { Escala de comunicación } \\
\text { relacional, Test de ajuste } \\
\text { marital }\end{array}$ \\
\hline $\begin{array}{l}\text { W a c k e r y } \\
\text { Dziobek }(2018)\end{array}$ & $\begin{array}{l}\text { C u a s i - } \\
\text { experimental } \\
\text { mixtor no } \\
\text { aleatorizados } \\
\text { con grupo } \\
\text { control }\end{array}$ & $\begin{array}{l}43 \text { profesionales } \\
\text { de medicina, } \\
\text { e n f e r m e ría y } \\
\text { administración (+ } \\
61 \text { grupo control) }\end{array}$ & $\begin{array}{l}3 \text { sesiones de } 7 \text { horas. } \\
\text { Observación, Sentimientos, } \\
\text { Necesidades, Peticiones, } \\
\text { Escucha empática }\end{array}$ & $\begin{array}{l}\text { Escala de CNV de } \\
\text { elaboración propia, Índice } \\
\text { de reactividad } \\
\text { interpersonal, Estrés } \\
\text { emocional percibido, } \\
\text { Escala de intensidad de } \\
\text { estrés laboral } \\
\text { Grupo de discusión }\end{array}$ \\
\hline
\end{tabular}

En el campo de las Ciencias de la Salud, los 4 estudios revisados proceden de EE.UU. y se basan en intervenciones formativas de $\mathrm{CNV}$ aplicadas en el ámbito penitenciario y de la profesión de enfermería.

Marlow, Nyamathi, Grajeda, Bailey, Weber y Younger (2012) midieron el impacto de una estrategia formativa de $\mathrm{CNV}$ en una muestra de 30 internos de programas de rehabilitación de adicciones (Tabla 1). Integrado en el paradigma de la salud mental en centros penitenciarios (Haney, 2003), el estudio reveló la utilidad de la $\mathrm{CNV}$ como abordaje terapéutico de las adicciones, y su eficacia para mejorar la comprensión del concepto de empatía, así como para la construcción de redes de apoyo en contextos de internamiento. En este caso, resultó relevante el relato de uno de los internos: "Lo que más me sorprendió es que la gente está pensando sus respuestas y consejos antes siquiera de acabar de escuchar. $\mathrm{Y}$ yo era una de esas personas. Ahora le doy la oportunidad a la gente de expresarse y yo sólo escucho y así puedo entender mejor o tener empatía con sus necesidades o sentimientos. Puedo verdaderamente mantener una conversación, no sólo "no tengo tiempo para charlar" o cosas así. Esto fue lo más impactante de la CNV para mí" (Marlow, Nyamathi, Grajeda, Bailey, Weber y Younger, 2012:13).

Desde el campo de la formación en Enfermería, Nosek y su equipo publicaron dos experiencias orientadas a mejorar la empatía en 
estudiantes de pregrado (Nosek, 2012; Nosek, Gifford y Kober, 2014). La primera asentó los fundamentos teóricos dentro de la ética de la autenticidad (Taylor, 1991) y pilotó la estrategia formativa en $\mathrm{CNV}$ con estudiantes de enfermería. La segunda mostró un incremento leve pero estadísticamente significativo de la reactividad personal en alumnado de enfermería (Tabla 1). En la evaluación cualitativa a los 2 años, la población participante identificaba la CNV como herramienta útil para conectar empáticamente con los pacientes, tal y como mostraba el testimonio de una enfermera: "Aprender comunicación noviolenta fue uno de las cosas más importantes que aprendí en el grado. La uso todos los días, varías veces incluso, tanto en mi vida personal como en el trabajo. Esas herramientas cambiaron completamente mi modo de interactuar con las personas y creo que cambiaron también cómo me ven las personas a mí, con confianza, amable y profesional" (Nosek, Gifford y Kober, 2014:11)

Por último, Boothe, Frasier, Waver y White-Kiehle (2018) presentaron en un estudio de caso, la aplicabilidad de la CNV para completar y facilitar la aplicación de dos modelos de resolución de conflicto -Conversaciones cruciales (Patterson et al, 2012) y Teamstepps (Peters et al, 2018), en el ámbito interprofesional hospitalario.

Dentro de las Ciencias Sociales, se identificaron 5 trabajos de Psicología y de 2 de Trabajo Social y Comunitario.

En el artículo de Suárez, Lee, Rowe, Gomez, Murowchick y Linn (2014), el equipo estadounidense midió, a través de dos estudios cuasi-experimentales, el impacto de la intervención formativa de CNV sobre la tasa de reincidencias (estudio 1), así como sobre las actitudes y conductas (estudio 2) en 4 centros penitenciarios (Tabla 1). La formación combinó sesiones de $\mathrm{CNV}$ con mindfulness (Kabat-Zinn, 2003) y redujo la tasa de reincidencias del $37 \%$ al $21 \%(p<0,05)$. La evaluación cualitativa con juegos de rol mostró el aumento de la autocompasión, expresión emocional y empatía, así como una disminución de la agresividad entre los internos participantes.

En Alemania, dos estudios evaluaron el impacto de la CNV sobre la empatía y salud emocional del personal sanitario. Wacker y Dziobek (2018) partieron de teorías explicativas en torno al estrés empático (Eisenberg 2000) y el trabajo emocional (Hochschild 1983) en el ámbito laboral (tabla 1). Tras evaluar la formación, los autores concluyeron que los principios de CNV mejoran la verbalización de emociones negativas y la comunicación colaborativa, a la vez que reducen el estrés empático. Si bien no se encontraron diferencias concluyentes en torno al impacto sobre la empatía cognitiva y emocional, la CNV sí se consideró una herramienta formativa eficiente para mejorar habilidades emocionales e interprofesionales y para prevenir el estrés en el ámbito sanitario.

Los también alemanes Altman, Schönefeld y Rorth (2015) evaluaron una estrategia formativa en $\mathrm{CNV}$, orientada a mejorar la empatía y prevenir el desajuste emocional en profesionales de enfermería en prácticas (Tabla 1). El fundamento teórico del estudio fue el modelo del corto circuito emocional (Altman y Roth, 2013) y los resultados cuantitativos mostraron un aumento significativo de los niveles de auto-percepción, auto-aceptación y claridad emocional, así como la mejora del desajuste emocional (irritación e implicación) y de la resiliencia.

En el continente asiático, el ámbito de la psicología proporcionó dos estudios cuasiexperimentales que usaron la $\mathrm{CNV}$ como estrategia formativa. El primero de ellos, iraní, 
trabajó con adolescentes de zonas desfavorecidas de Teheran, y evaluó el impacto de la CNV en los estilos de afrontamiento y la tendencia suicida (Nafise y Ghazal, 2018). Realizado en el marco teórico-conceptual sobre estilos de afrontamiento y estrés (Endler y Parker, 1999), el estudio mostró que la CNV reduce la intención de suicidio, genera la adopción del afrontamiento emocional eficaz y mejora las habilidades de resolución de conflictos entre jóvenes en riesgo de exclusión social.

Desde Filipinas, Vazhappilly y Reyes (2017) propusieron el estudio de la comunicación como base para la calidad de las relaciones de pareja (Troy, 2000). En su estudio realizado con parejas, utilizaron la escala de comunicación relacional y el test de ajuste marital, para concluir que la formación en CNV representa una herramienta psicoterapéutica eficiente para mejorar la calidad y satisfacción dentro de la relación conyugal.

En el ámbito del Trabajo social y comunitario, se identificaron 2 estudios que usaron la formación en CNV en la atención socio-comunitaria a poblaciones vulnerables, ambos orientados a reducir la violencia $y$ mejorar la convivencia étnica.

Nosek y Durán (2017) evaluaron el impacto de la CNV en las habilidades de empatía y resolución de conflictos en personas latinas residentes en EE.UU. Las autoras revelaron la satisfacción y gratitud de las personas participantes, así como el impacto positivo de la formación en la capacidad empática, la conciencia y actitud de cambio, los vínculos comunitarios y la comprensión emocional intra e inter-personal.

Por otra parte, en Turquía, Alshughry (2018) realizó una intervención comunitaria con mujeres sirias de un centro de acogida para personas refugiadas. Para ello, usó el modelo teórico Mayor-menor (Patfoort, 1995) para explicar los mecanismos de violencia y lo aplicó a nivel práctico a través de las técnicas de Teatro del Oprimido (Boal, 2005). En su estudio de caso, encontró que la CNV facilita la identificación emocional, mejora la definición y gestión de situaciones de conflicto y representa una herramienta de fácil aplicabilidad en la vida diaria.

Desde la perspectiva multidisciplinar, se identificaron 2 estudios.

El primero, canadiense (Museux, Dumont, Careau y Milot, 2016), evaluó una estrategia formativa dirigida a profesionales de asistencia socio-sanitaria. El trabajo se realizó desde el marco de competencia interprofesional (CIHC 2010) y de diversos modelos de mejora de la comunicación interprofesional (Haig, Sutton y Whittington, 2006; Reeves et al., 2003). La formación en CNV ayudó a crear un lenguaje interprofesional común, clarificó la definición de roles, mejoró el enfoque centrado en el cliente, aunque mostró escaso impacto en la comunicación interprofesional y el liderazgo colaborativo. Por otra parte, la satisfacción del personal participante fue alta, por la originalidad, componente práctico e impacto positivo en la conciencia y empatía.

Burgon, DiGammage y Hebden (2017) combinaron la perspectiva de Trabajo Social, Psico-pedagogía y Ciencias del deporte para aplicar la CNV a la psico-terapia basada en la equitación. Una vez situado el marco teórico del aprendizaje basado en la equitación asistida (Bachi, 2012), las autoras presentaron una etnografía de la experiencia con adolescentes de 10-15 años con diversas afecciones emocionales. La CNV se consideró un marco teórico adecuado para abordar los aspectos relacionales y mejorar la interacción afectiva entre actores humanos y no humanos participantes en la terapia.

La Figura 5 organiza los 13 trabajos realizados en el campo de las Ciencias de la 
Salud, las Ciencias Sociales y el ámbito multidisciplinar, esquematizando la información referente a: procedencia académica de autores y revistas, poblaciones diana y principales dimensiones y resultados de la aplicación e impacto de la CNV.

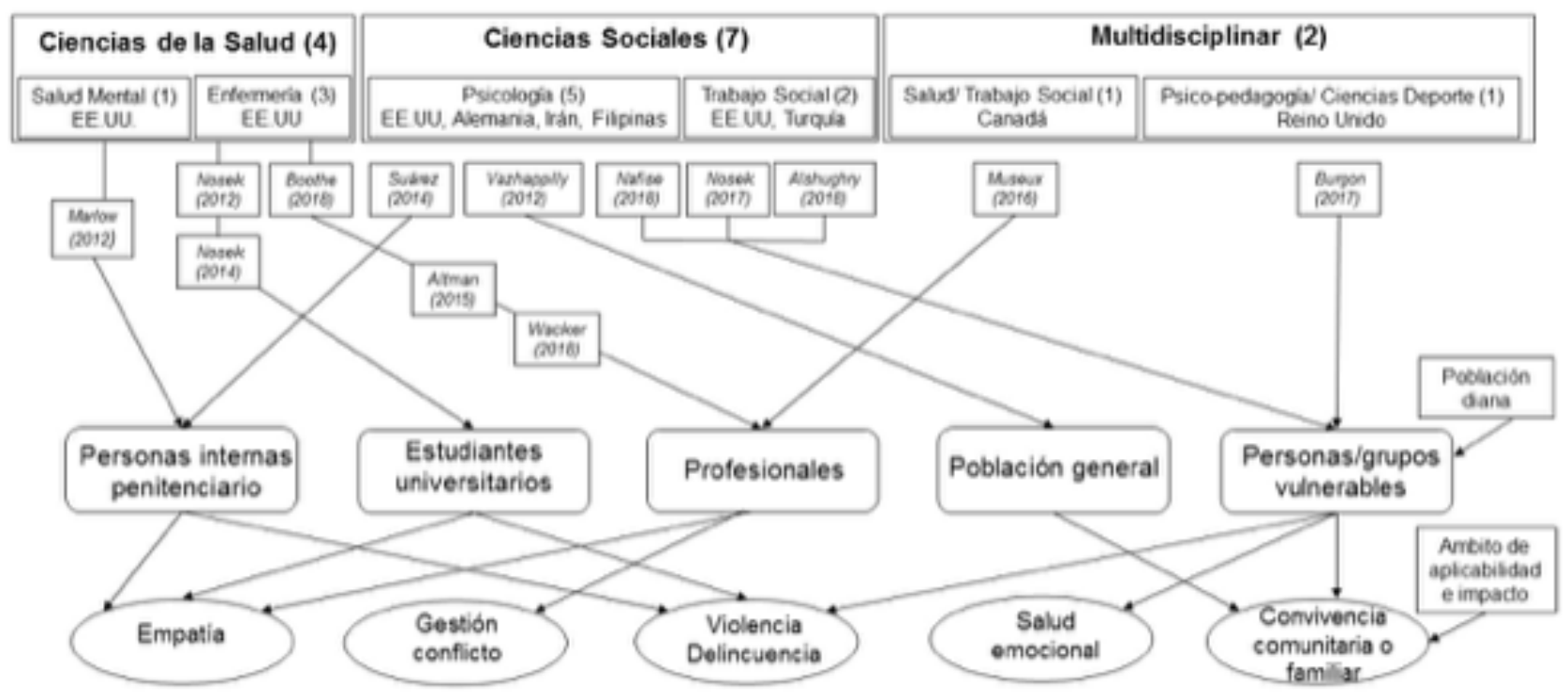

FIGURA 5. LA CNV EN CIENCIAS DE LA SALUD, CIENCIAS SOCIALES Y ENFOQUE MULTIDISCIPLINAR: POBLACIONES DIANA Y DIMENSIONES DE IMPACTO.

\section{Discusión y conclusiones}

Esta revisión pone de manifiesto el creciente interés de la comunidad científica por la CNV y su aplicación a nivel internacional. Los 23 estudios educativos, sanitarios, sociales y multidisciplinares evaluaron teórica o empíricamente los beneficios de la CNV en docencia e investigación. La distribución geográfica mundial de los estudios es dispersa, con mayor concentración en el continente norteamericano, donde la CNV representa una línea consolidada en algunos grupos de trabajo.

Desde las Ciencias de la Educación, la construcción y debate en torno a marcos teóricos para la aplicación de la CNV alcanza especial relevancia en EE.UU. y Canadá, donde la mayoría de los artículos son ensayísticos. Por otra parte, las experiencias de Europa y Asia son estudios descriptivos, de diseño y pilotaje de estrategias formativas específicas $\mathrm{y}$, en estos contextos, la propia
CNV se contempla como un marco conceptual en sí.

Desde la Pedagogía y Didáctica, la CNV se integra teóricamente como herramienta eficaz para el desarrollo del pensamiento crítico-reflexivo, orientado a la acción $y$ justicia social, lo que también se ha explorado desde modelos de educación intercultural (Mikolic, 2017) Por otra parte, se debate también la conexión de la CNV con la espiritualidad y la paz, desde un doble enfoque: como vivencia interiorizada y desde el activismo pacifista.

Pese a la heterogeneidad de perspectivas teóricas y prácticas, la $\mathrm{CNV}$ se considera unánimemente un paradigma útil para crear comunidades educativas inclusivas, fomentar la integración y el enfoque colaborativo en las aulas, a la vez que aumentar el nivel de empatía y mejorar la expresión emocional (Stueck, 2010). La Figura 4 evidencia el consenso total con respecto a los beneficios de la CNV sobre el desarrollo de competencias 
inter-personales. Entre ellas, se fundamentan las habilidades y aptitudes para la interacción, comunicación (escucha y expresión) y las de carácter ético o aplicables en contextos socioculturales: el respeto, la inclusión y compromiso social y comunitario. Por otra parte, la mejora de competencias intrapersonales está documentada sólo en 6 de los 10 estudios revisados, lo que verifica la descripción de la revisión koreanas (Yang y Kim, 2016).

En los estudios de Ciencias de la Salud, los resultados revelan el impacto de la formación en $\mathrm{CNV}$ sobre los niveles de empatía en profesionales sanitarios (Chung y Kim, 2011). Este resultado adquiere especial importancia en vistas de que la comunicación empática en el encuentro asistencial genera mayor precisión diagnóstica, más adherencia a tratamientos y mejores resultados en salud, a la vez que es determinante en la toma de decisiones compartida (Orsini, 2018).

Por otra parte, una revisión sistemática sobre estrategias empáticas en estudiantes de enfermería (Everson, Levett-Jones y Pitt, 2018), encuentra que el diseño de los estudios, incluyendo la intervención con CNV de Nosek (2014), tiene en general baja calidad metodológica, lo que se puede aplicar a los estudios revisados aquí.

En el ámbito académico psico-social y multidisciplinar, se confirma la prevalencia de estudios cuasi-experimentales (Yang y Kim, 2016), algunos con intervención sin grupo control y no se identifica ningún enfoque longitudinal. La estructura y contenidos de las estrategias formativas y las poblaciones diana son altamente heterogéneas y los instrumentos de evaluación son dispares, lo que complica la presentación de resultados concluyentes en torno al impacto de la CNV a partir de los estudios revisados. Sin embargo, quedan avalados los beneficios de la CNV sobre la empatía, gestión del conflicto, prevención y abordaje de la violencia y delincuencia, así como sobre aspectos psicosociales, de salud emocional y calidad de relaciones familiares, interprofesionales y comunitarias (Burleson, Martin y Lewis, 2011; Eckstein y La Grassa, 2005; Yang y Kim, 2016; Romeo-Biedma, 2014).

Por último, este trabajo tiene una serie de limitaciones. Pese al método sistemático de revisión, el sesgo idiomático y de indexación de las revistas, pudo dejar artículos sin revisar. El procedimiento de evaluación cualitativa y síntesis narrativa, la heterogeneidad de temáticas, ámbitos, metodologías y contextos geográficos y socio-culturales, dificultó la comparación de resultados. Sin embargo, las fortalezas de la revisión son la originalidad y espectro amplio de análisis, que permitió sintetizar en un solo documento resultados provenientes de varios ámbitos científicoacadémicos.

Esta revisión pone de manifiesto la heterogeneidad de perspectivas y abordajes disciplinares y metodológicos en la aplicación y evaluación de la CNV en el ámbito científico-académico. La CNV se integra en el marco teórico humanista $\mathrm{y}$, a nivel práctico, genera el desarrollo de competencias y mejoras en las vivencias personales, la interacción y convivencia entre individuos, promoviendo actitudes y comportamientos empáticos en ámbitos educativos, familiares, laborales $\mathrm{y}$ comunitarios.

Como propuestas para el futuro, sería necesario fomentar los diseños metodológicos sólidos, que aporten, desde ámbitos científicos y académicos, resultados basados en la evidencia sobre el impacto de la CNV y su aplicación. 


\section{Referencias Bibliográficas}

Agnew, Elizabeth N. (2012) Needs and Nonviolent Communication in the Religious Studies Classroom, Teaching Theology and Religion, v. 15, $\mathrm{n}^{\mathrm{o}} 3$, pp. 210-224.

Albe, Virginie y Gombert, María José (2012) Students' communication, argumentation and knowledge in a citizens' conference on global warming. Cult Stud of Sci Educ, v. 7, pp. 659-681. DOI: https://doi.org/10.1007/s11422-012-9407-1

Alshughry, Usama (2018) Non-violent communication and theatre of the oppressed : a case study with Syrian refugee women from the Kareemat Centre in Turkey. Intervention, v. 16, $\mathrm{n}^{\mathrm{o}} 2$, 170-174. DOI: https://doi.org/10.4103/INTV.INTV

Altmann, Tobias y Roth, Marcus (2013) The evolution of Empathy: from single components to process models, en C. Mohiyeddini, M. Eysenck, S. Bauer (Eds.), Handbook of Psychology of Emotions. New York, Nova Science Publishers, pp. 171-188.

Altmann, Tobias, Schönefeld, Victoria y Roth, Marcus (2015) Evaluation of an Empathy Training Program to Prevent Emotional Maladjustment Symptoms in Social Professions. Psychology, v. 6, pp. 1893-1904.

Bachi, Keren (2012) Equine-facilitated psychoteraphy: the gap between practice and knowledge. Society and Animals, v. 20, pp. 364-380.

Baesler, E. James (2017) I' d Rather Teach Peace : An Autoethnographic Account of the Nonviolent Communication and Peace Course. Communication \& Theatre Arts Faculty Publications, v. 16, pp. 71-75.

Baesler, E. James y Lauricella, Sharon (2014) Teach peace : assessing instruction of the nonviolent communication and peace course. Journal of Peace Education, v. 11, n ${ }^{\circ}$ 1, pp. 46-63. DOI: https://doi.org/10.1080/17400201.2013.777899

Bandura, Albert (1973) Aggression: A social learning analysis. Prentice Hall, New Jersey, Englewood Cliffs.

Bernstein, Basil (1996) El dispositivo pedagógico en pedagogía, control simbólico e identidad. Madrid, Morata.

Bisquerra, Rafael (2003) Educación emocional y competencias básicas para la vida. Revista de Investigación Educativa, 21. [En línea] https://revistas.um.es/rie/article/view/99071/94661 [consultado el 10 de junio de 2019].

Boal, Augusto (2002) Games for actors and non-actors. London, Routledge.

Booth, Wayne (2004) The Rhetoric of Rhetoric: the Quest for Effective Communication. Malden, MA, Wiley- Blackwell.

Boothe, Amy, Frasier, Nora, Weaver, Christy y White-Kiehle, Jessica (2018) Resolving Conflict: what does the giraffe say? Nurse Leader, v. 16, $\mathrm{n}^{\mathrm{o}} 2$, 121-126. DOI: https://doi.org/ 10.1016/j.mnl.2017.10.008

Bourdieu, Pierre (1999) Understanding, en P. Bourdieu y G. Balazs (Eds.). The weight of the world: social suffering in contemporary society, Stanford, Stanford University Press, pp. 607-626.

Burbules, Nicholas y Rice, Suzanne (1991) Dialogue across differences: continuing the conversation. Harvard Educational Review, v. 61, pp. 393-416. 
Burgon, Hannah, Gammage, Di y Hebden, Jenny (2017). Hoofbeats and heartbeats : equineassisted therapy and learning with young people with psychosocial issues - theory and practice, Journal of Social Work Practice. DOI: https://doi.org/10.1080/02650533.2017.1300878

Burgoon, Judee K. y Hale, Jerold E. (1987) Validation and measurement of the fundamental themes of relational communication. Communication Monographs, v. 54, no. 1, pp. 19-41.

Burleson, Molly, Martin, Monique y Lewis, Rashunda (2011) Assessing the Impact of Nonviolent Communication. The Center for Nonviolent Communication [En línea] https:// www.cnvc.org/sites/cnvc.org/files/NVC_Research_Files/ [Consultado el 25 de enero de 2019]

Chopp, Rebecca (2015) Loving the future: interview with Rebecca Chopp. Religious Studies News, v. 4, pp. 5-10.

Chung, Hee-Shim y Kim, Soon-Lae (2011) Effects of a Nonviolent Communication Program for Hospital Nurse Managers in Korea. Paper presented at: Sigma Theta Tau 22nd International Nursing Research Congress, Cancun, Mexico.

CIHC (Canadian Interprofessional Health Collaborative). (2010) A national interprofessional competency framework. Vancouver, Canada, Canadian Interprofessional Health Collaborative.

Connor, Jane M. y Wentworth, Robert (2012). Training in Collaborative Communication in an Organizational Context: Assessment of Impact, en Psychologists for Social Responsibility 30th Anniversary Conference, Washington DC.

Cox, Elaine y Dannahy, Patricia (2005) The value of openness in e-relationships: Using nonviolent communication to guide online coaching and mentoring. International Journal of Evidence Based Coaching and Mentoring, v. 3, n 1, pp. 39-51.

D'Amour, Danielle y Oandasan, Ivy (2005) Interprofessionality as the field of interprofessional practice and interprofessional education: An emerging concept. Journal of Interprofessional Care, v. 19, S8-S20.

Davis, Mark H. (1980). A multidimensional approach to individual differences in empathy. JSAS Catalog of Selected Documents in Psychology, v. 10, p. 85.

Dougan, Debbie (2011) Can you see the beauty? Nonviolent communication as a counter narrative in the lives of former prisoners (Doctoral dissertation, Washington State University). Dissertation Abstracts International, 71(10A), 3613.

Dzaferovic, Mirsada (2012). Nonviolent Communication, empathy and assertion as modern strategies in the study of overcoming conflicts. Metodicki Obzori, v. 7, pp. 105-116.

Eckstein, Daniel y La Grassa, Lucy (2005) The Non-Violent Relationship Questionnaire (NVRQ). The Family Journal: Counseling and Therapy for couples and families, v. 13, nº. 2, pp. 205-211. DOI: https://doi.org/10.1177/1066480704273134

Eisenberg, Nancy (2000) Emotion, regulation, and moral development. Annual Review of Psychology, v. 51, pp. 665-697. DOI: https://dx.doi.org/10.1146/annurev .psych.51.1.665

Endler, Norman S y Parker, James D. (1999) Coping Inventory for Stressful Situations (CISS): Manual. 2nd Edition, Multi-Health Systems, Toronto.

Everson, Naleya, Levett-Jones, Tracy y Pitt, Victoria (2018). The impact of educational interventions on the empathic concern of health professional students: A literature review. Nurse Education in Practice, 5. DOI: https://doi.org/10.1016/j.nepr.2018.05.01 
Fassett, Deanna L. y Warren, John T. (2005) The strategic Rhetoric of an "At-risk" Educational Identity: interviewing Jane. Communication and Critical/cultural Studies, v. 2, pp. 238-256.

Finfgel-Connett, Deborah (2014) Use of content analysis to conduct knowledge-building and theory-generating qualitative systematic reviews. Qualitative Research, v. 14, nº 3, pp. 341-352.

Freire, Paulo (2004) Pedagogy of indignation. Paradigm Publishers, Boulder, London.

Frey, Renea (2018) Rhetorics of Reflection: Revisiting Listening Rhetoric through Mindfulness, Empathy, and Non-violent Communication. JAEPL, v. 23, n 247, pp. 92-104.

Gall, Meredith D; Gall, Joyce P. y Borg Walter R. (2003) Educational Research: An Introduction. Seventh edition. United States, Pearson Education, Inc.

Gandhi, Mahatma K. (1971) Non-violence, en J. G. Murphy (Ed.), Civil disobedience and violence. Belmont, CA, Wadsworth, pp. 93-102.

Gannett, Cinthia y Brereton, John C. (Eds.). (2016) Traditions of Eloquence: the Jesuits and Modern Rhetorical Studies. New York, Fordham UP.

Haig, Kathleen M; Sutton, Staci y Whittington, John (2006) SBAR: A shared mental model for improving communication between clinicians. Joint Commission Journal on Quality and Safety, v. $32, n^{\circ} .6$, pp. 167-175.

Haney, Craig (2003). Mental health issues in long-term solitarity confinement and supermax confinement. Crime and delinquency, v. 49, pp. 125-156.

Hao, Richie Neil (2011) Critical Compassionate Pedagogy and the Teacher's Role in FirstGeneration Student Success. NEW DIRECTIONS FOR TEACHING AND LEARNING, v. 127, pp. 91-98. DOI: https://doi.org/10.1002/tl

Hochschild, Arlie R. (1983) The managed heart. Berkeley, CA, University of California.

Hooper, Lee (2015) An exploratory study: Nonviolent communication strategies for secondary teachers using a Quality Learning Circle approach. [MA Thesis]. University of Canterbury, Christchurch. [En línea] https://www.cnvc.org/sites/default/files/research/ Lee Hooper_Master's\%20Thesis.pdf [consultado el 10 de junio de 2019].

Jones, Suzanne (2009). Traditional Education or Partnership Education: Which Educational Approach Might Best Prepare Students for the Future? (Unpublished Master's Thesis). San Diego University, San Diego, CA.

Kabat-Zinn, Jon (2003) Mindfulness-based interventions in context: Past, present, and future. Clinical Psychology: Science and Practice, v. 10, pp. 144-156. DOI: https://doi:10.1093/clipsy/ $\underline{\operatorname{bpg} 016}$

Koegel, Rob (2002) Nonviolent communication and partnership education. Encounter: Education for Meaning and social justice, v. 15, $\mathrm{n}^{\mathrm{o}} 3$, pp. 2-4.

Kök, Havva. (2009). Nonviolent communication in political conflicts. USAK Yearbook Internat Politics and Law, v. 2, pp. 349-62. [En línea] http://www.usak.org.tr/images upload/files/ makale17 2009.pdf [consultado el 10 de junio de 2019].

Leu, Lucy (2003) Nonviolent communication companion workbook. Encinitas, CA, Puddle Dance Press.

López Martínez, Mario (2015) Nonviolence in social sciences: towards a consensual definition. Revista de Paz y Conflictos, v. 8, pp. 63-81. 
López Martínez, Mario (2000) La sociedad civil por la paz, en Muñóz, A. y López Martínez, M. Historia de la paz. Tiempos, espacios y actores. Granada: Ed. Universidad de Granada, pp. 291-357.

MacIntyre, Alasdair (2006) Toleration and the Goods of Conflict, en Ethics and Politics: selected essays. Cambridge: Cambridge University Press, pp. 205-223.

Madrigal Villegas, Vera y Vargas Barquero, Vivian (2014) Implementación de estrategias de la comunicación no violenta y la educación emocional en el aprendizaje de un segundo idioma. Revista de Lenguas Modernas, v. 20, pp. 323-334.

Marlow, Elizabeth, Nyamathi, Adeline, Grajeda, William T; Bailey, Newt, Weber, Amanda y Younger, Jerry (2012) Nonviolent Communication Training and Empathy in Male Parolees. Journal of Correctional Health Care, v. 18, $\mathrm{n}^{\circ}$. 1, pp. 8-19. DOI: https://doi.org/ $\underline{10.1177 / 1078345811420979}$

McCarthy, Colman (2002) I'd rather teach peace. New York, NY, Orbis Books. Matters.

Mercer, Neil (1996) The guided construction of knowledge. Clevedon, England, Multilingual

Mikolic, Vesna (2016) Ethnic identity and intercultural awareness in modern language teaching: Tilka model for ethnic conflicts avoidance. Hauppauge, New York, Nova Science Publishers.

Museux, Anne- Claire, Dumont, Serge, Careau, Emmanuelle y Milot, Elise (2016) Improving interprofessional collaboration: The effect of training in nonviolent communication Improving. Social Work in Health Care. DOI: https://doi.org/10.1080/00981389.2016.1164270

Nafise, Heshmati-Molaie y Ghazal, Zandkarimi (2018) Non-Violent Communication and Its Impact on Suicidal Ideations and Coping with Stress Styles. Journal of Behavioral and Brain Science, v. 8, pp. 147-156. DOI: https://doi.org/10.4236/jbbs.2018.84009

Nash, Andrea L. (2007) Case Study of Tekoa Institute: Illustration of Nonviolent communication Training's Effect on Conflict Resolution. MS Sociology. Virginia Polytechnic Institute and State University, Blacksburg.

Nosek, Marcianna (2012) Nonviolent communication: A dialogical retrieval of the ethic of authenticity. Nursing Ethics, v. 19, nº, pp. 829-837.

Nosek, Marcianna y Durán, Maite (2017) Increasing Empathy and Conflict Resolution Skills through Nonviolent Communication (NVC) Training in Latino Adults and Youth. Progress in Community Health Partnership: Research, Education and Action, v. 11, n 3, pp. 275-283.

Nosek, Marciana, Gifford, Ekizabeth y Kober, Brendan (2014) Nonviolent Communication (NVC) training increases empathy in baccalaureate nursing students: A mixed method study. Journal of Nursing Education and Practice, v. 4, no 10, pp. 1-15. DOI: https://doi.org/10.5430/ jnep.v4n10p1

Orsini, Anthony J. (2018) Get With the PROGRAM: A Guide to Compassionate Communication. Journal American Osteopathy Association, v. 118, n ${ }^{\circ}$ 10, pp. 679-684. DOI: https://doi.org/10.7556/jaoa.2018.149

Patfoort, Pat (1995) Uprooting violence building nonviolence: from nonviolent upbridging to a nonviolence society. Freeport, MA, Cobblesmith.

Patterson, Kerry, Grenny, Joseph, McMillian, Ron Switzer, Al (2012) Crucial Conversations: Tools for Talking when the Stakes are High. New York, NY, McGraw-Hill. 
Peters, V.Kristen, Harvey, Ellen M., Wright, Andi, Bath, Jennifer, Freeman, Dan y Collier, Brian. (2018). Impact of a TeamSTEPPS trauma nurse academy at a level 1 trauma center. J Emerg Nurs, v. 44, pp. 19-25. hppts://dx.doi.org/10.1016/j.jen.2017.05.007

Qudsyi, Hazhira, Trimulyaningsih, Nita, Novitasari, Resnia y Stueck, Marcus (2018) Developing module of nonviolent communication among children in Yogyakarta, en International Conference on Child-Friendly Education, pp. 149-160.

Ratcliffe, Krista (2005). Rhetorical Listening: identification, Gender, Whiteness. Carbondale, IL: Southern Illinois.

Reeves, Scott, Russel, Ann, Zwarenstein, Merrick, Kenaszchuk, Chris., Conn, Lesley. G., Doran, Diane et al. (2003) Structuring communication relationships for interprofessional teamwork (SCRIPT): A Canadian initiative aimed at improving patient-centred care. Journal of Interprofessional Care, v. 21, $\mathrm{n}^{\mathrm{o}} 1$, pp. 11-114.

Romeo-Biedma, F. Xavier (2014) Crossing focusing and Nonviolent Communication. The Folio. A Journal for Focusing and Experiential Therapy, v. 25, n ${ }^{\circ}$ 1, pp. 111-123.

Rosenberg, Marshall B. y Molho, Pascale (1998) Nonviolent (empathic) communication for health care providers. Haemophilia, v. 4, pp. 335-340.

Rosenberg, Marshall B. (2003) Nonviolent communication: a language of life. Encinitas, CA, Puddledancer Press.

Rosenberg, Marshall B. y Chopra, Deepak (2015). Nonviolent communication: a language of life: life-changing tools for healthy relationships. Encinitas, CA, Puddledancer Press.

Schwind, Jasna K., McGay, Elizabeth, Mertersky, Kateryna, y Martin, Jennifer (2016). Development and Implementation of an Advanced Therapeutic Communication Course: An Interprofessional Collaboration. J Nurs Educ, v. 55, no 10, pp. 592-597. DOI: https://doi.org/ $\underline{10.3928 / 01484834-20160914-11}$

Schwind, Jasna K. (2016) Narrative reflective process: A creative experiential path to personal-knowing in teaching-learning scholarship, en J. Gingras, P. Robinson, J. Waddell, y L.D. Cooper (Eds.), Teaching as scholarship: Preparing students for professional practice in community services. Waterloo, ON, Wilfrid Laurier University Press, pp. 137-154.

Sears, Melanie W. (2013) Non violent communication: Application to health care (Unpublished doctoral dissertion). Kahjlui, Hawaii: University of Hawaii Maui College. [En línea] https://www.cnvc.org/about-us/projects/nvc-research [consultado el 10 de junio de 2019].

Shamseer, Larissa, Moher, David, Clarke, Mike, Ghersi, Davina, Liberati, Alessandro y Petticrew, Mark (2015) Preferred reporting items for systematic review and meta-analysis protocols (PRISMA-P). Elaboration and explanation. British Medical Journal, v. 2, pp. 349.

Shoemaker, Dan J. (2004). Non-violent communication as a treatment for inmates. Virginia Polytechnic Institute\& State University, DHHS Public Health Services.

Stueck, Marcus. (2010). Introduction in the concept of the School of Empathy based on the integrated model of empathy. Material education for instructor in Indonesia. Center Educational Health Germany.

Suarez, Alejandra, Lee, Dug Y., Rowe, Cristopher, Gomez, Alex A., Murowchick, Elise y Linn, Patricia L. (2014) Freedom Project : Nonviolent Communication and Mindfulness Training in Prison. SAGE Open. DOI: https://doi.org/10.1177/2158244013516154 
Taylor Charles. The ethics of authenticity. (1991). Cambridge, MA, Harvard University Press, 1991.

Tong, Allison, Flemming, Kate, McInnes, Elizabeth, Oliver, Sandy y Craig, Jonathan (2012). Enhancing transparency in reporting the synthesis of qualitative research: ENTREQ. BMC Medical Research Methodology, v. 12, pp. 181.

Torres Morales, Maribel (2013). La violencia en los escenarios educativos. Cuaderno de Investigación En La Educación, v. 28, pp. 91-108.

Troy, Adam B. (2000) Determining the factors of intimate relationship satisfaction: Interpersonal communication, sexual communication and communication affect. Colgate University Journal of Science, v. 32, pp. 221-230.

Vazhappilly, Joshy J. y Reyes, Marc E. S. (2017) Non-Violent Communication and Marital Relationship: Efficacy of Emotion-Focused Couples Communication Program among Filipino Couples. Psychological Studies. DOI: https://doi.org/10.1007/s12646-017-0420-z

Vygotsky, Lev S. (1982). Obras Escogidas: Tomos I y II. (J. M. Bravo, Trad.). Madrid: Visor Distribuciones.

Wacker, Renata y Dziobek, Isabel (2018). Preventing Empathic Distress and Social Stressors at Work Through Nonviolent Communication Training: A Field Study With Health Professionals. Journal of Occupational Health Psychology, v. 23, n. 1, pp. 141-150. DOI: https://doi.org/10.1037/ $\underline{\text { ocp0000058 }}$

Younis, Jaklein R., Mabrouk, Sohair M y Kamal, Fawzia F. (2015) Effect of the planned therapeutic communication program on therapeutic communication skills of pediatric nurses. Journal of Nursing Education and Practice, v. 5, pp. 109-120. DOI: https://doi:10.5430/ jnep.v5n8p109

PROCESO EDITORIAL • EDITORIAL PROCESS INFO

Recibido: 12/06/2019 Aceptado: 14/05/2020

CÓMO CITAR ESTE ARTÍCULO • HOW TO CITE THIS PAPER

Danet Danet, Alina (2020). La comunicación noviolenta entre teoría y práctica. Una revisión sistemática, Vol.13 (1), 35-55.

\section{SOBRE LOS AUTORES • ABOUT THE AUTHORS}

Alina Danet Danet es doctora de Sociología (Universidad de Granada) y actualmente profesora en la Universidad de Jaén. Sus principales líneas de investigación, con metodología cuantitativa y cualitativa, se integran en el ámbito de la comunicación, género y salud, aspectos emocionales de los procesos salud-enfermedad y la relación entre profesionales sanitarios y ciudadanía. 